\title{
Where does signet-ring cell carcinoma come from and where does it go?
}

\author{
Tomio Arai ${ }^{1}$
}

Received: 17 March 2019 / Accepted: 20 March 2019 / Published online: 8 April 2019

(c) The International Gastric Cancer Association and The Japanese Gastric Cancer Association 2019

Signet-ring cell (SRC) carcinoma, named for its resemblance to a signet ring, once was called "mucocellular adenocarcinoma". SRC carcinoma is a unique name for a specific adenocarcinoma, the cells of which show a large amount of mucin in the cytoplasm that displaces the nucleus to the cell periphery.

According to the current World Health Organization (WHO) classification defined in 2010 [1], SRC carcinoma is not an independent histological subtype, but it is included in the poorly cohesive (PC) carcinoma subtype. Recently, interest in reviewing the classification of PC and SRC carcinomas is increasing. Among the histological classifications of gastric cancer (GC), both SRC and mucinous carcinomas differ in definition from other subtypes, such as tubular and papillary adenocarcinomas. Rather than the degree of formation of tubular and papillary structures, SRC and mucinous carcinomas are classified based on a signet-ring-like morphology or a large amount of mucus production, respectively. In a recent issue of Gastric Cancer, Mariette et al., on behalf of the European Chapter of the International Gastric Cancer Association, presented a consensus on the pathological definition and classification of PC GC, including SRC carcinoma of the stomach [2].

This editorial thus focuses on the relationship between SRC and PC carcinomas and reviews the WHO classification in comparison with the Japanese classification. SRC carcinoma was classified as one of the histological subtypes of GC in the 1st [3], 2nd [4], and 3rd [5] editions of the WHO classification. However, SRC carcinoma now is part of the PC carcinomas in the current classification [1]. SRC carcinoma can easily transform into PC carcinoma, especially in invasive areas. Additionally, it is often difficult to

Tomio Arai

arai@tmig.or.jp

1 Department of Pathology, Tokyo Metropolitan Geriatric Hospital and Institute of Gerontology, 35-2 Sakaecho, Itabashi-ku, Tokyo 173-0015, Japan differentially diagnose the tumor as SRC or PC carcinoma. Thus, the current classification seems to be reasonable in a certain context.

Professor Kunio Oota, the first president of our institute, was deeply involved in both the 1st edition (1977) of the WHO classification [3] and the 9th edition (1974) of the Japanese classification, in which the SRC carcinoma was first described [6]. In fact, the 1st edition of the WHO classification was largely the same as the 9th edition of the Japanese classification. Because the main editors of the 1st and 2nd editions were then replaced by European and American researchers in the 3rd edition, minor differences occurred between the WHO and Japanese classifications. In the 4th edition of the WHO classification, gastric adenocarcinoma with little glandular differentiation was reclassified as PC carcinoma based on an emphasis on mutual adhesiveness of tumor cells. On the other hand, the current Japanese classification [7], the 15th edition 2017, largely follows the 9th edition. Regarding the differences, since the 12th edition (1993) of the Japanese classification, poorly differentiated adenocarcinoma (PDA) has been divided into the subtypes solid and non-solid, because tumors with relatively better prognosis exist in PDAs. The non-solid type PDA corresponds to a histological subtype of the PC carcinoma [1]. In contrast, the solid-type PDA shows characteristic clinicopathological and biological features, typical genetic alterations, and a better prognosis. The solid-type PDA is almost equivalent to a solid carcinoma as per the definition and characterization in the WHO classification. Our recent study provided evidence that tumors of this histological subtype show a high frequency of microsatellite instability and accumulate in the gastric antrum, especially in older women [8]. Thus, more attention should be paid to this subtype because it could be a target tumor that is treatable with immune checkpoint blockade [9].

In the 1st edition of the WHO classification, SRC carcinoma was defined as a tumor composed predominantly or exclusively of signet-ring cells characterized by a central, 
optically clear, globoid droplet of cytoplasmic mucin with an eccentrically placed nucleus. However, it did not show a single characteristic morphology. At least three kinds of SRC carcinoma cells were described in the 1 st edition of the WHO classification: (1) a cell with an intracytoplasmic cyst filled with acid mucin, giving the classical signet-ring appearance; (2) a tumor cell with eosinophilic cytoplasmic granules containing neutral mucin with a slightly eccentric nucleus; and (3) a tumor cell in which the cytoplasm is distended, with secretory granules of acid mucin appearing like a goblet cell. These three cell types may occur independently or in combination within any tumor. This tumor tends to infiltrate diffusely and is often associated with considerable fibrosis ("scirrhous"). Pure SRC carcinoma is relatively rare and it is usually present in the early stage, especially in the intramucosal layer. Its morphology is often lost during tumor growth and transformation into PC carcinoma. In the 3rd edition of the WHO classification, small and anaplastic cells with little or no mucin were also classified as SRC carcinoma; however, these cell types were reclassified as PC carcinoma in the 4th edition. As Mariette et al. recently proposed the usage of the terminologies SRC and PC carcinomas according to their proportion within the tumor [2], we should reconsider the relationship between SRC and PC carcinomas. Some SRC and PC carcinomas share common biological behaviors and can be recognized as the same disease entity. Although the WHO classification emphasizes the mutual cohesiveness of tumor cells, we may need to pay more attention to cellular differentiation.

Considering the mucin type, the 1st edition of the WHO classification described two SRC carcinoma types, with neutral mucus and acid mucilage (goblet cell SRC carcinoma) [3]. Recent advances in mucin immunohistochemistry and molecular biology allowed for the examination of SRC carcinoma in detail. However, only a few reports discussed a relationship between the mucin expression profile and gastric SRC and PC carcinomas [10, 11]. Genetic alterations in GC have been comprehensively analyzed, and the results provided a novel classification [12]. SRC and PC carcinomas are now classified according to histology, mucin phenotype, and specific protein expression. However, these tumors will be genetically classified in the near future.

In SRC carcinoma, some tumors extend to the intramucosal layer without vertical invasion, reaching more than $10 \mathrm{~cm}$ in diameter. However, some SRC carcinomas tend to invade the submucosal layer even at the early stage. Currently, we cannot differentiate between them using biopsy specimens collected before treatment initiation. If differentiation were possible, indications for endoscopic therapy could be extended to SRC carcinomas without vertical invasion.

Overall, poorly differentiated adenocarcinomas such as SRC, PC, and solid carcinomas should be examined using the latest research methods. This would have significant implications for daily clinical practice.

\section{References}

1. Lauwers GY, Carneiro F, Graham DY, Curado MP, Franceschi S, Montgomery E, et al. Tumours of the stomach. In: Bosman FT, Carneiro F, Hruban RH, Theise ND, editors. World Health Organization classification of tumours of the digestive system. 4th ed. Lyon: International Agency for Research on Cancer; 2010. p. 45-58.

2. Mariette C, Carneiro F, Grabsch HI, van der Post RS, Allum W, de Manzoni G, et al. Consensus on the pathological definition and classification of poorly cohesive gastric carcinoma. Gastric Cancer. 2019;22:1-9.

3. Oota K, Sobin LH. Histological typing of gastric and oesophageal tumours. Geneva: World Health Organization; 1977.

4. Watanabe H, Jass JR, Sobin LH. Histological typing of oesophageal and gastric tumours. 2nd ed. Berlin: Springer; 1990.

5. Fenoglio-Preiser C, Carneiro F, Correa P, Guiford P, Lambert R, Megraud F, et al. Tumours of the stomach. In: Hamilton SR, Aaltone LA, editors. World Health Organization classification of tumours pathology and genetics of tumours of the digestive system. 3rd ed. Lyon: IARC Press; 2000. p. 37-56.

6. Japanese Gastric Cancer Association. Japanese classification of gastric cancer. 9th ed. Tokyo: Kanehara; 1974 (in Japanese).

7. Japanese Gastric Cancer Association. Japanese classification of gastric carcinoma. 15th ed. Tokyo: Kanehara; 2017 (in Japanese).

8. Arai T, Matsuda Y, Aida J, Takubo K, Ishiwata T. Solid-type poorly differentiated adenocarcinoma of the stomach: clinicopathological and molecular characteristics and histogenesis. Gastric Cancer. 2019;22:314-22.

9. Le DT, Uram JN, Wang H, Bartlett BR, Kemberling H, Eyring $\mathrm{AD}$, et al. PD-1 blockade in tumors with mismatch-repair deficiency. N Engl J Med. 2015;372:2509-20.

10. Kwon CH, Kim YK, Lee S, Kim A, Park HJ, Choi Y, et al. Gastric poorly cohesive carcinoma: a correlative study of mutational signatures and prognostic significance based on histopathological subtypes. Histopathology. 2018;72:556-68.

11. Tian MM, Zhao AL, Li ZW, Li JY. Phenotypic classification of gastric signet ring cell carcinoma and its relationship with clinicopathologic parameters and prognosis. World J Gastroenterol. 2007;13:3189-98.

12. Cancer Genome Atlas Research Network. Comprehensive molecular characterization of gastric adenocarcinoma. Nature. 2014;513:202-9.

Publisher's Note Springer Nature remains neutral with regard to jurisdictional claims in published maps and institutional affiliations. 